\title{
GAIA Level 1 Intra-abdominal Pregnancy
}

National Cancer Institute

\section{Source}

National Cancer Institute. GAIA Level 1 Intra-abdominal Pregnancy. NCI Thesaurus. Code C128751.

GAIA Level 1 Intra-abdominal Pregnancy is defined by the discovery of a fetus within the abdominal cavity during laparotomy, without evidence of uterine rupture, and with placentation not within the uterine cavity. 